\title{
Start low, go slow, but don't go this way yet
}

\author{
Brian Bressler MD MS FRCPC, Robert Enns MD
}

$\mathrm{A}$ zathioprine (AZA) and 6-mercaptopurine (6-MP) are critical medications with proven benefits and an acceptable safety profile, used in both Crohn's disease and ulcerative colitis (1-4). Understanding the metabolism of AZA/6-MP has created insight into the efficacy and side effects associated with it (5-9). The prodrug AZA is metabolized to 6-MP. 6-MP is then metabolized by one of three enzymes (Figure 1). Xanthine oxidase converts 6-MP to 6-thiouric acid. Thiopurine methyltransferase (TPMT) and hypoxanthine phosphoribosyl transferase metabolize 6-MP to various metabolites, including 6-methylmercaptopurine and 6-thioguanine (6-TGN).
The two major limitations of AZA/6-MP are the duration of time needed to achieve clinical efficacy, and the toxicities and intolerance associated with them. Because AZA/6-MP is important for treating patients with inflammatory bowel disease, much research has focused on how to achieve optimal clinical efficacy while minimizing complications (6,10-16). As recommended by the US Food and Drug Administration, some gastroenterologists are using pharmacogenetics to identify patients with specific genetic mutations that result in low TPMT enzymatic activity, which may predict hematological toxicity from AZA/6-MP (17-22). Following the metabolites

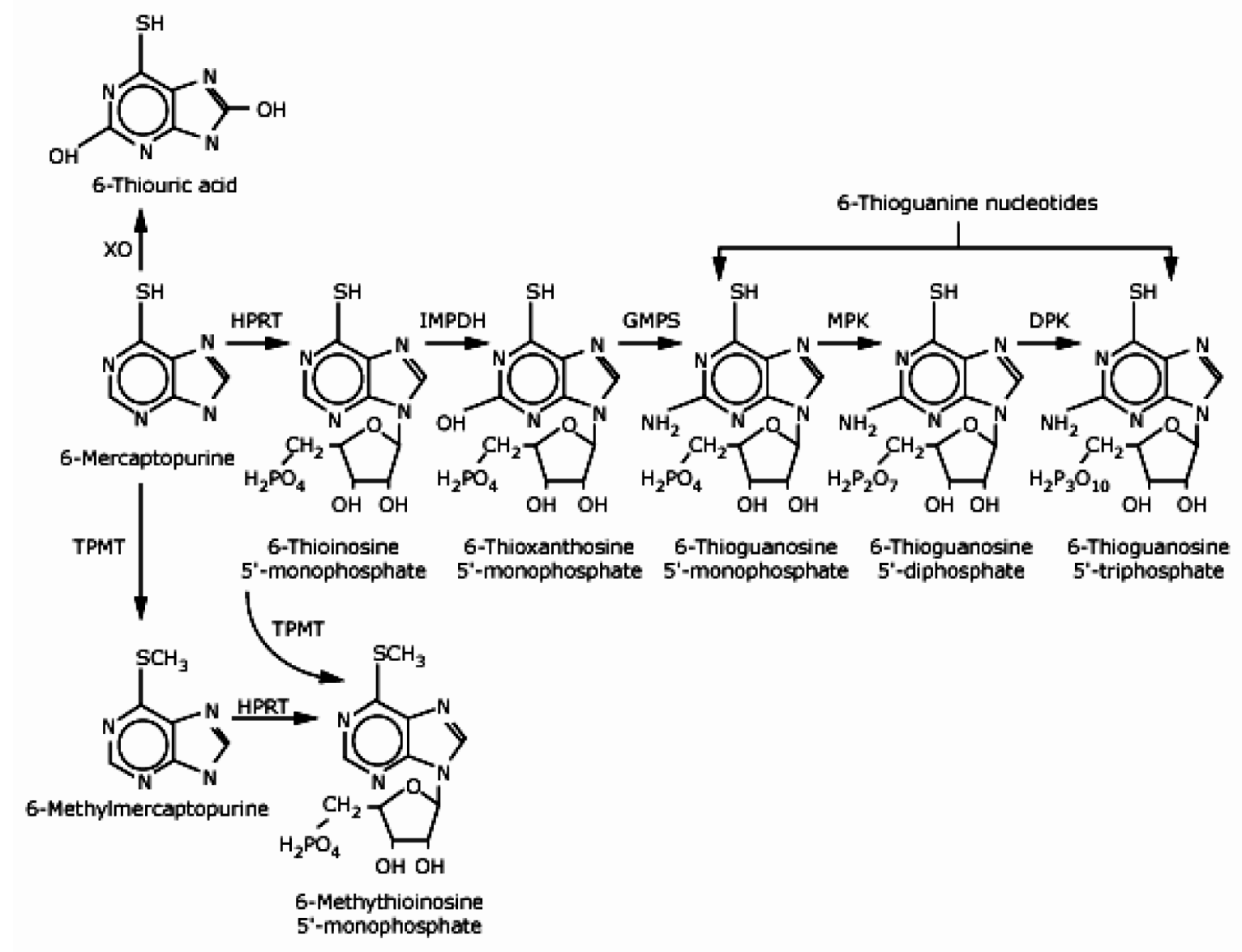

Figure 1) The metabolism of 6-mercaptopurine (6-MP). DPK Diphosphate kinase; GMPS Guanine monophosphate synthetase; HPRT Hypoxanthine phosphoribosyl transferase; IMPDH Inosine monophosphate dehydrogenase; MPK Monophosphate kinase; TPMT Thiopurine methyltransferase; XO Xanthine oxidase. Reproduced with permission from reference 28

Division of Gastroenterology, University of British Columbia, Vancouver, British Columbia

Correspondence: Dr Brian Bressler, Division of Gastroenterology, University of British Columbia, 770-1190 Hornby Street, Vancouver, British Columbia V6Z 2K5. Telephone 604-68-6332, fax 604-689-2004, e-mail brian_bressler@hotmail.com

Received and accepted for publication January 25, 2008 
of AZA/6-MP may predict potential hepatotoxicity (6-methylmercaptopurine [6-MMPN] levels $5700 \mathrm{pmol} / 8 \times 10^{8}$ red blood cells [RBC] or higher) and the likelihood of clinical efficacy (6-TGN levels $230 \mathrm{pmol} / 8 \times 10^{8} \mathrm{RBC}$ or higher). To avoid unnecessary toxicity in patients with TPMT deficiency and to achieve optimal drug dosing, many centres will measure both the genotype and 6-TGN levels $(9,23,24)$. The absence of prospective randomized trials addressing the utility of metabolite testing in patients on AZA/6-MP, the limited availability of these tests and the cost of these investigations are the major reasons why Canadian gastroenterologists are not routinely using these tests to optimize management of patients with inflammatory bowel disease on AZA/6-MP. If the first limitation is addressed, then the other two factors limiting our use of AZA/6-MP should be a priority that is undertaken to overcome these issues in Canada.

In other countries, such as the United States, metabolite testing is more readily used, because availability is less of an issue and private medical insurance companies reimburse for the expense of these tests. Following metabolites results of patients using AZA/6-MP has commonly identified individuals on presumably adequate doses of these medications according to their weight, but who have subtherapeutic levels of 6-TGN. Once confirmed that patients are actually taking the prescribed doses of AZA/6-MP, the dose of these drugs can be increased with the intent of achieving therapeutic 6-TGN levels without causing toxicity. In certain patients, because of rapid metabolism, therapeutic levels of 6-TGN cannot be obtained because of the associated hepatotoxic risk AZA/6-MP can theoretically cause at high doses. In this issue of The Canadian Journal of Gastroenterology, Witte and Ginsberg (pages 181-85) report on using lower doses of 6-MP combined with allopurinol in four patients unable to reach therapeutic 6-TGN levels because of hepatoxicity. The ability of allopurinol to raise 6-TGN levels and lower 6-MMPN levels, thereby improving disease activity and normalizing liver enzymes, has been previously reported to be possible $(25,26)$. Witte and Ginsberg have described the potential influence of allopurinol on AZA/6-MP metabolism. The net effect of using allopurinol with AZA/6-MP is to potentially increase the efficacy of this medication by increasing 6-TGN levels, but also increasing the risk of bone marrow suppression. The case series raises caution in contemplating such an approach. The first case highlights one of the controversies surrounding the use of metabolite monitoring in that although therapeutic levels of 6-TGN and normal liver enzymes were achieved by using low-dose 6-MP and allopurinol, there did not seem to be any clinical benefit after this manipulation. Unfortunately, the successful increase in 6-TGN levels likely did contribute to the patient developing infectious complications - facial herpes zoster as a consequence of leukopenia. The second case also demonstrates the significant risk of developing leukopenia with this strategy, although the patient did not develop an infectious complication. Furthermore, this patient apparently achieved clinical remission with a 6-TGN level of only $222 \mathrm{pmol} / 8 \times 10^{8} \mathrm{RBC}-$ lower than the level that is thought to be required to reach this end point. However, high levels of 6-MMPN do not always lead to hepatoxicity, as was shown in the third case. Without documented elevated liver enzymes, manipulating AZA/6-MP to lower 6-MMPN levels is not thought to be necessary. Therefore, increasing the patient's 6-MP dose without using allopurinol may have led to a similar clinical end point without exposing the patient to an increased risk of leukopenia. The final case shows proof of both the biochemical and clinical efficacies of using low-dose 6-MP with allopurinol.

The authors should be commended for reporting on their use of low-dose 6-MP with allopurinol, because this will add to our collective experience with the utility of using metabolite 6-MP testing, and the impact of manipulating AZA/6-MP metabolism. Clearly, this approach should not be routinely used in Canada for many reasons. First, metabolite testing is not commonly being used in usual clinical practice in this country, and without access to these tests, this strategy would not be safe. The use of allopurinol in itself needs to be studied further, because the mechanism of why allopurinol increases 6-TGN levels is unclear. Most importantly, the utility of targeting AZA/6-MP doses based on their metabolite levels must be studied in a prospective fashion before embarking on the strategy of manipulating the metabolism of AZA/6-MP to achieve an enhanced clinical response, with increased potential toxicity.

There are two primary methods used in Canada to initiate AZA/6-MP: 'start low and go slow' and 'start immediately with a presumed therapeutic dose with or without TPMT genotyping and enzyme activity testing'. Gastroenterologists who start low and gradually escalate argue that this may be safer and better tolerated, particularly if the patient is genetically susceptible to toxicity at low doses. Gastroenterologists who start with a full dose argue that the toxicity will be present at a low or high dose, and adherence to routine hematological testing is required to discontinue the drug if toxicity is noted. Once patients are on AZA/6-MP, some physicians dose these medications based on the patient's body weight, while others escalate the dose until leukopenia or increased mean corpuscular volume is achieved $(8,24,27)$. Until the optimal way to start and escalate the dose of AZA/6-MP is determined, these approaches seem reasonable, but we would caution against using allopurinol in the way described in the case series presented by Witte and Ginsberg until further evidence clarifies the clinical efficacy and potential toxicity.

\section{REFERENCES}

1. Cuffari C. How useful is thiopurine metabolite monitoring in pediatric inflammatory bowel disease? Nat Clin Pract Gastroenterol Hepatol 2008;5:12-3.

2. Dubinsky MC, Reyes E, Ofman J, Chiou CF, Wade S, Sandborn WJ. A cost-effectiveness analysis of alternative disease management strategies in patients with Crohn's disease treated with azathioprine or 6-mercaptopurine. Am J Gastroenterol 2005;100:2239-47.

3. Dubinsky MC. Azathioprine, 6-mercaptopurine in inflammatory bowel disease: Pharmacology, efficacy, and safety. Clin Gastroenterol Hepatol 2004;2:731-43.

4. Su C, Lichtenstein GR. Treatment of inflammatory bowel disease with azathioprine and 6-mercaptopurine. Gastroenterol Clin North Am 2004;33:209-34, viii.

5. Winter J, Walker A, Shapiro D, Gaffney D, Spooner RJ, Mills PR. Cost-effectiveness of thiopurine methyltransferase genotype screening in patients about to commence azathioprine therapy for treatment of inflammatory bowel disease. Aliment Pharmacol Ther 2004;20:593-9.

6. Black AJ, McLeod HL, Capell HA, et al. Thiopurine methyltransferase genotype predicts therapy-limiting severe toxicity from azathioprine. Ann Intern Med 1998;129:716-8.

7. Gisbert JP, Nino P, Rodrigo L, Cara C, Guijarro LG. Thiopurine methyltransferase (TPMT) activity and adverse effects of azathioprine in inflammatory bowel disease: Long-term follow-up study of 394 patients. Am J Gastroenterol 2006;101:2769-76.

8. Decaux G, Prospert F, Horsmans Y, Desager JP. Relationship between red cell mean corpuscular volume and 6-thioguanine 
nucleotides in patients treated with azathioprine. J Lab Clin Med 2000;135:256-62.

9. Goldenberg BA, Rawsthorne P, Bernstein CN. The utility of 6-thioguanine metabolite levels in managing patients with inflammatory bowel disease. Am J Gastroenterol 2004;99:1744-8.

10. Lowry PW, Franklin CL, Weaver AL, et al. Leucopenia resulting from a drug interaction between azathioprine or 6-mercaptopurine and mesalamine, sulphasalazine, or balsalazide. Gut 2001;49:656-64.

11. Dubinsky MC, Vasiliauskas EA, Singh H, et al. 6-thioguanine can cause serious liver injury in inflammatory bowel disease patients. Gastroenterology 2003;125:298-303.

12. Colombel JF, Ferrari N, Debuysere H, et al. Genotypic analysis of thiopurine S-methyltransferase in patients with Crohn's disease and severe myelosuppression during azathioprine therapy. Gastroenterology 2000;118:1025-30.

13. Gilissen LP, Derijks LJ, Driessen A, et al. Toxicity of 6-thioguanine: No hepatotoxicity in a series of IBD patients treated with long-term, low dose 6-thioguanine. Some evidence for dose or metabolite level dependent effects? Dig Liver Dis 2007;39:156-9.

14. de Boer NK, Derijks LJ, Gilissen LP, et al. On tolerability and safety of a maintenance treatment with 6-thioguanine in azathioprine or 6 mercaptopurine intolerant IBD patients. World J Gastroenterol 2005;11:5540-4.

15. Winter JW, Gaffney D, Shapiro D, et al. Assessment of thiopurine methyltransferase enzyme activity is superior to genotype in predicting myelosuppression following azathioprine therapy in patients with inflammatory bowel disease. Aliment Pharmacol Ther 2007;25:1069-77

16. Roblin X, Becot F, Jacquot JM, Nairi A, Abinder J, Monnet D. [Azathioprine-induced acute pancreatitis]. Ann Gastroenterol Hepatol (Paris) 1990;26:233.

17. Lennard L, Chew TS, Lilleyman JS. Human thiopurine methyltransferase activity varies with red blood cell age. Br J Clin Pharmacol 2001;52:539-46.

18. Lennard L, Lilleyman JS. Individualizing therapy with 6-mercaptopurine and 6-thioguanine related to the thiopurine methyltransferase genetic polymorphism. Ther Drug Monit 1996;18:328-34.

19. Cuffari C, Dassopoulos T, Turnbough L, Thompson RE, Bayless TM. Thiopurine methyltransferase activity influences clinical response to azathioprine in inflammatory bowel disease. Clin Gastroenterol Hepatol 2004;2:410-7.

20. Dubinsky MC, Lamothe S, Yang HY, et al. Pharmacogenomics and metabolite measurement for 6-mercaptopurine therapy in inflammatory bowel disease. Gastroenterology 2000;118:705-13.

21. Spire-Vayron de la Moureyre C, Debuysere H, Mastain B, et al. Genotypic and phenotypic analysis of the polymorphic thiopurine Smethyltransferase gene (TPMT) in a European population. Br J Pharmacol 1998;125:879-87.

22. Andersen JB, Szumlanski C, Weinshilboum RM, Schmiegelow K. Pharmacokinetics, dose adjustments, and 6-mercaptopurine/ methotrexate drug interactions in two patients with thiopurine methyltransferase deficiency. Acta Paediatr 1998;87:108-11.

23. Derijks LJ, de Jong DJ, Gilissen LP, et al. 6-Thioguanine seems promising in azathioprine- or 6-mercaptopurine-intolerant inflammatory bowel disease patients: A short-term safety assessment. Eur J Gastroenterol Hepatol 2003;15:63-7.

24. Belaiche J, Desager JP, Horsmans Y, Louis E. Therapeutic drug monitoring of azathioprine and 6-mercaptopurine metabolites in Crohn disease. Scand J Gastroenterol 2001;36:71-6.

25. Sparrow MP, Hande SA, Friedman S, Cao D, Hanauer SB. Effect of allopurinol on clinical outcomes in inflammatory bowel disease nonresponders to azathioprine or 6-mercaptopurine. Clin Gastroenterol Hepatol 2007;5:209-214.

26. Sparrow MP, Hande SA, Friedman S, et al. Allopurinol safely and effectively optimizes thioguanine metabolites in inflammatory bowel disease patients not responding to azathioprine and mercaptopurine. Aliment Pharmacol Ther 2005;22:441-6.

27. Lichtenstein GR. Use of laboratory testing to guide 6-mercaptopurine/ azathioprine therapy. Gastroenterology 2004;127:1558-64.

28. Cuffari C, Theoret Y, Latour S, Seidman G. 6-Mercaptopurine metabolism in Crohn's disease: Correlation with efficacy and toxicity. Gut 1996;39:401-6. (Erratum in 1998;43;301). 


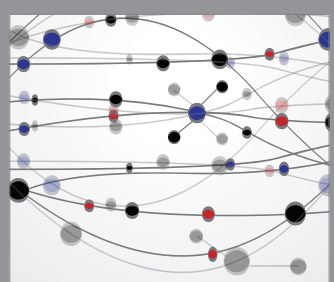

The Scientific World Journal
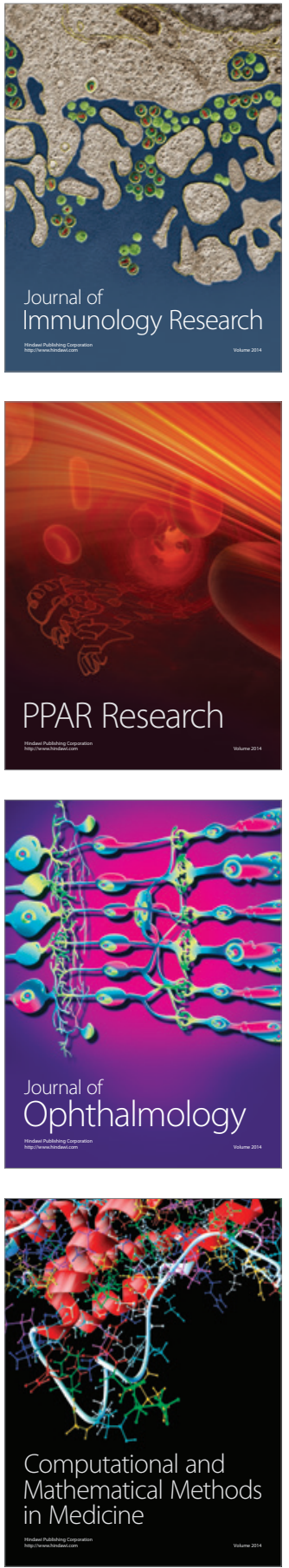

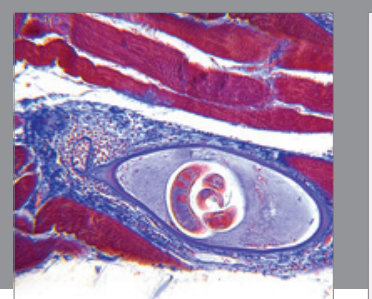

Gastroenterology Research and Practice

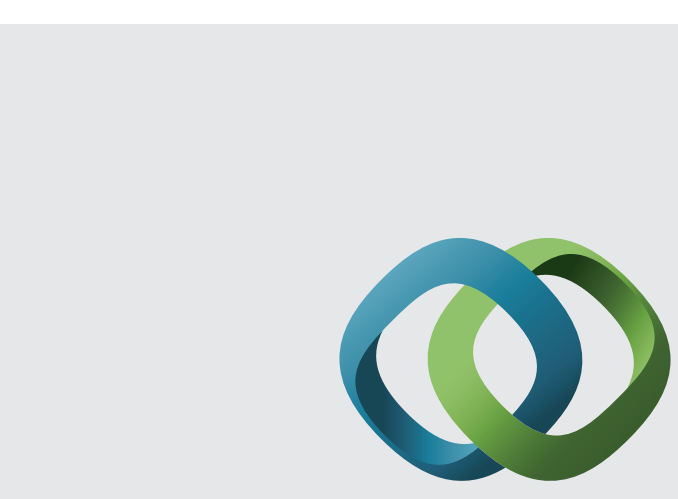

\section{Hindawi}

Submit your manuscripts at

http://www.hindawi.com
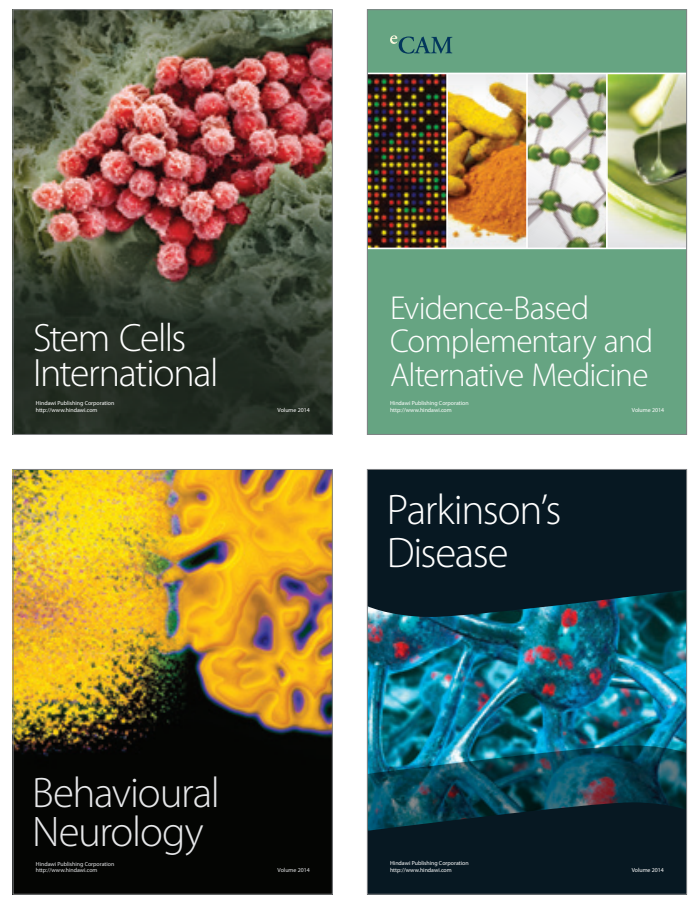
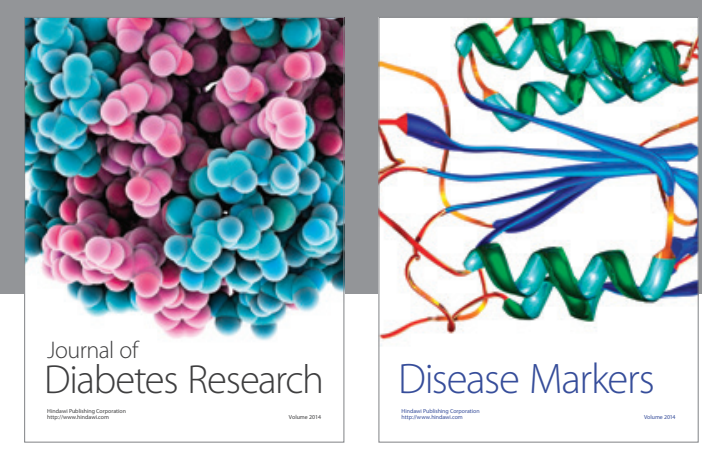

Disease Markers
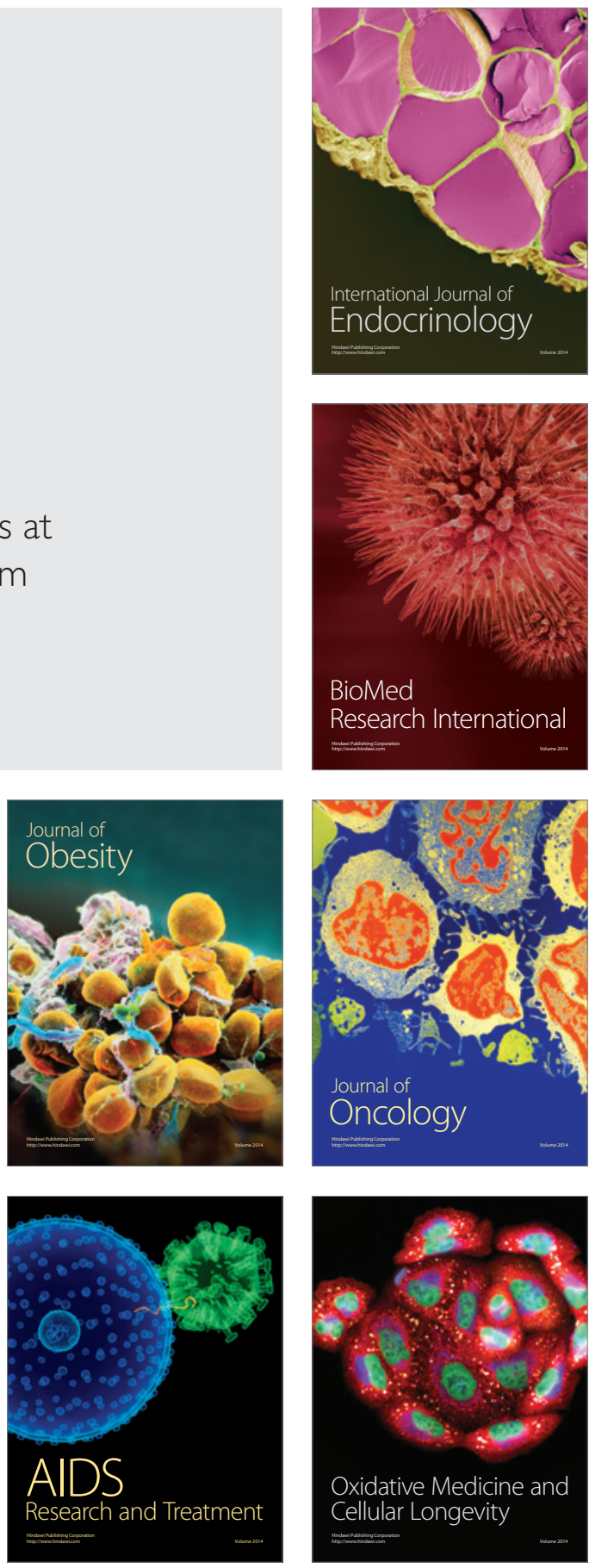